\title{
Long and short high energy components presented in GRBs
}

\author{
N. Fraija*, M. M. Gonzalez, R. Sacahui and W. H. Lee \\ Instituto de Astronomia, UNAM, Mexico, 04510, Universidad Nacional Autónoma de México, \\ Circuito Exterior, C.U., A. Postal 70-264, 04510 Mexico D.F., Mexico \\ nifraijaeastro.unam.mx, magdadastro.unam.mx, \\ jsacahuidastro.unam.mx, wleedastro.unam.mx
}

\begin{abstract}
We present a leptonic model on the external shock framework to describe the long- and shortlasting GeV component of some GRBs. This model was already applied successfully to GRB 090926A, and we extend it to describe the high-energy emission of GRB 090902B and GRB 090510. We argue that the high-energy emission consists of two components, one at $\mathrm{MeV}$ energies with a duration of a few seconds during the prompt phase, and a second $\mathrm{GeV}$ component lasting hundred of seconds after the prompt phase. The short high-energy component can be described as SSC emission from a reverse shock and the longer component arises from SSC emission of the forward shock. The main assumption of our model is that the jet is magnetized and evolves in the thick-shell case. The calculated fluxes and break energies are all consistent with the observed values
\end{abstract}

Gamma-Ray Bursts 2012 Conference-GRB2012,

May 07-11, 2012

Munich, Germany

\footnotetext{
*Speaker.
} 
Hadronic [1, 2] and leptonic [3, 4] models of GRBs have been widely discussed to explain photons with energies $\geq 100 \mathrm{MeV}$. In particular, Fraija et al. (2012) and Sacahui et al. (2012) showed that the short $\mathrm{MeV}$ and long-lasting GeV high-energy components presented in GRB 980923 and GRB 090926A respectively, could come from SSC emission in external shocks. We apply the same model in GRB 090902B and GRB 090510 and show that only the assumption of a magnetized jet is required to explain the complete high-energy emission. Introducing standard values for the input parameters, we obtain break energies, fluxes, duration, etc in agreement with the observed values. A brief description of the high-energy emission to described for the considered bursts is given below.

GRB 090510 is a short burst observed by both GBM and LAT instruments on board of the FERMI mission[5]. In the first minute post-trigger, 191 events with energy above $100 \mathrm{MeV}$ and 30 events with energy above $1 \mathrm{GeV}$ were detected by LAT. A photon with energy $30.5_{-2.6}^{+5.8} \mathrm{GeV}$ was detected by LAT $0.829 \mathrm{~s}$ after the GBM trigger and an extra power law component with power law function of photon index $-1.62 \pm 0.03$ was used to describe the high-energy emission. The onset of the high energy spectral component with respect to the beginning of the prompt emission is at $\sim$ $0.1 \mathrm{~s}$. We are taking into account the photons above $100 \mathrm{MeV}$ starting at $\sim T_{0}+0.8 \mathrm{~s}$.

GRB 090902B is a long burst observed by GBM and LAT instruments [6] at the J2000 coordinates $\mathrm{RA}=265^{\circ}$ and $\mathrm{Dec}=27.33^{\circ}$. This burst was detected significantly by LAT with 39 photons with energy above $1 \mathrm{GeV}$. High energy emission from this burst was detected up to $\mathrm{E}=33.4_{-3.5}^{+2.7}$ $\mathrm{GeV}$ at $82 \mathrm{~s}$ after the GBM trigger. Photons were detected as late as $300 \mathrm{~s}$ after the trigger. The time-integrated spectrum of GRB 090902B is best modeled by a Band function and a power law component with an index of $-2.1 \pm 0.1$ and its flux declines as $t^{-1.5 \pm 0.1}$ over the time interval $\left(T_{0}\right.$ $+25, T_{0}+1000 \mathrm{~s}$ ). We are taking into account the photons above $100 \mathrm{MeV}$ starting at $\sim T_{0}+13 \mathrm{~s}$.

GRB 090926A is a long burst observed by Fermi LAT/GBM [7] from the J2000 coordinates $\mathrm{RA}=354.5^{\circ}$ and $\mathrm{Dec}=-64.2^{\circ}$. GRB 090926A presents a distinct high energy power law component [7] separate from the known BAND function, fitted by a Band+CUTPL function with a high energy spectral break at $\mathrm{E}_{f}=-1.41_{-0.42}^{+0.22} \mathrm{GeV}$ and with a power law index of $-1.72 \pm_{-0.02}^{+0.10}$. A short episode in coincidence with a sharp spike apparent in the light curve above $100 \mathrm{MeV}$ at $\sim T_{0}+10 \mathrm{~s}$ is described as a power law with a cutoff energy. The flux between $10 \mathrm{keV}$ and $10 \mathrm{GeV}$ for this short episode is $22.29 \pm 1.60 \times 10^{-6} \mathrm{erg} \mathrm{cm}^{-2} \mathrm{~s}^{-1}$ and the power law index is $\lambda=-1.71_{-0.05}^{+0.02}$.

\section{Leptonic Model (Forward and Reverse shocks)}

In the external shock model, GRB emission is produced when an expanding relativistic shell interacts with the circumburst medium producing forward and reverse shocks. In the forward shock, electrons are accelerated to a power law distribution through the first Fermi mechanism. Thus, $N\left(\gamma_{e}\right) d \gamma_{e} \propto \gamma_{e}^{-p} d \gamma_{e}$, with $\gamma_{e} \geq \gamma_{m}$ and $\gamma_{m}=\varepsilon_{e, f}(p-2) /(p-1) m_{p} / m_{e} \gamma_{f}$, where $\varepsilon_{B, f}=$ $B_{f}^{2} /\left(32 \pi \gamma_{f}^{2} \eta_{f} m_{p}\right)$ and $\varepsilon_{e, f}=U_{e} /\left(4 \gamma_{f}^{2} \eta_{f} m_{p}\right)$ are the magnetic and electron equipartition parameters respectively, $\gamma_{f}$ is the Lorentz factor of the bulk and $\eta_{f}$ is the ISM density. Given the cooling electron Lorentz factor and the deceleration time, the break energies of the photons radiated by electrons at a distance $D$ from the source in natural units are given by, 


$$
\begin{aligned}
E_{\mathrm{m}, \mathrm{f}} & \sim \frac{2^{5 / 2} \pi^{1 / 2} q_{e} m_{p}^{5 / 2}(p-2)^{2}}{m_{e}^{3}(p-1)^{2}}(1+z)^{-1} \varepsilon_{e, f}^{2} \varepsilon_{B, f}^{1 / 2} n_{f}^{1 / 2} \gamma_{f}^{4} \\
E_{\mathrm{c}, \mathrm{f}} & \sim \frac{\pi^{7 / 6} 3^{4 / 3} m_{e} q_{e}}{2^{13 / 6} m_{p}^{5 / 6} \sigma_{T}^{2}}(1+z)^{-1}\left(1+x_{f}\right)^{-2} \varepsilon_{B, f}^{-3 / 2} n_{f}^{-5 / 6} E^{-2 / 3} \gamma_{f}^{4 / 3}
\end{aligned}
$$

where $E$ is the isotropic energy. The SSC breaks energies $\left(E_{m, f}^{(\mathrm{IC})} \sim \gamma_{m}^{2}, E_{m, f}\right.$ and $\left.E_{c, f}^{(I C)} \sim \gamma_{c}^{2} E_{c, f}\right)$ are also given by[8],

$$
\begin{aligned}
E_{\mathrm{m}, \mathrm{f}}^{(I C)} & \sim \frac{6 q_{e} m_{p}^{15 / 4}}{2^{5 / 4}(3 \pi)^{1 / 4} m_{e}^{5}}(1+z)^{5 / 4} \varepsilon_{e, f}^{4} \varepsilon_{B, f}^{1 / 2} n_{f}^{-1 / 4} E^{3 / 4} t_{f}^{-9 / 4} \\
E_{\mathrm{c}, \mathrm{f}}^{(I C)} & \sim \frac{2^{3 / 4} 27 \pi^{7 / 4} q_{e} m_{e}^{3}}{1283^{1 / 4} m_{p}^{9 / 4} \sigma_{T}^{4}}(1+z)^{-3 / 4}\left(1+x_{f}\right)^{-4} \varepsilon_{B, f}^{-7 / 2} n_{f}^{-9 / 4} E^{-5 / 4} t_{f}^{-1 / 4}
\end{aligned}
$$

On the other hand, when the reverse shock crosses the shell it heats up and accelerates electrons. Considering the thick shell case, when the ejecta is significantly decelerated, the synchrotron and SSC break energies are given by $[9,10]$,

$$
\begin{aligned}
E_{\mathrm{m}, \mathrm{r}} & \sim \frac{4 \pi^{1 / 2} q_{e} m_{p}^{5 / 2}(p-2)^{2}}{m_{e}^{3}(p-1)^{2}}(1+z)^{-1} \varepsilon_{e, r}^{2} \varepsilon_{B, r}^{1 / 2} \Gamma_{r}^{2} n_{r}^{1 / 2} \\
E_{\mathrm{c}, \mathrm{r}} & \sim \frac{9 \pi 2^{1 / 2} m_{e} q_{e}}{8\left(3^{1 / 2}\right) m_{p} \sigma_{T}^{2}}(1+z)^{-1 / 2}\left(1+x_{r}+x_{r}^{2}\right)^{-2} \varepsilon_{B, r}^{-3 / 2} n_{r}^{-1} E^{-1 / 2} T_{90}^{-1 / 2} \\
E_{\mathrm{m}, \mathrm{r}}^{(I C)} & \sim \frac{2^{21 / 4} \pi^{3 / 4} m_{p}^{13 / 4}(p-2)^{4}}{3^{1 / 4} m_{e}^{5}(p-1)^{4}}(1+z)^{-7 / 4} \varepsilon_{e, r}^{4} \varepsilon_{B, r}^{1 / 2} \Gamma_{r}^{4} n_{r}^{3 / 4} E^{-1 / 4} T_{90}^{3 / 4} \\
E_{\mathrm{c}, \mathrm{r}}^{(I C)} & \sim \frac{3^{7 / 2} \pi m_{e}^{3} q_{e}}{2^{11} m_{p}^{3} \sigma_{T}^{4}}(1+z)^{3 / 2}\left(1+x+x^{2}\right)^{-4} \varepsilon_{B, r}^{-7 / 2} n_{r}^{-3} E^{-1 / 2} \Gamma_{r}^{-6} T_{90}^{-5 / 2}
\end{aligned}
$$

where $T_{90}$ is the burst duration. A detailed description of the model is given in Fraija et al. (2012) and Sacahui et al. (2012).

\section{Results and Conclusions}

We have used typical $[9,10,11]$ values for $\varepsilon_{B, r} \sim 10^{-1}, \varepsilon_{B, f} \sim 10^{-4}, \eta_{f} \sim 10^{1} \mathrm{~cm}^{-3}, \varepsilon_{e, r} \sim 0.6$, $\varepsilon_{e, f} \sim 10^{-2}, \gamma_{f} \sim 600$ and $\gamma_{r} \sim 1000$. The calculated and observed quantities are given in Table 1.

To account for the shorter duration, as compared with the burst duration, of the high-energy emission at energies of hundreds of $\mathrm{MeV}$ it is required a value of $\varepsilon_{B, r} \sim 10^{-1}$, implying a highly magnetized jet. Moreover, a long-lived keV emission from synchrotron radiation in the forward shock is expected. Some of these bursts present a long-lived tail [12] which is interpreted as early afterglow. In other hand, the duration of the long-lived $\mathrm{GeV}$ component is calculated as the time when $E_{f, m}^{I C}$ drops below $\sim \mathrm{GeV}$, obtaining a value of $\sim 100 \mathrm{~s}$, in agreement with the observations. 
In summary, we have presented a leptonic model based on external shocks to describe the long GeV- and short MeV- emission in a unified manner for GRB 090926A, GRB 090510 and GRB 090902B. The main requirement is a magnetized ejecta.

\begin{tabular}{|c|c|c|c|}
\hline GRBs & 090510 & 090902B & 090926A \\
\hline \multicolumn{4}{|l|}{ Forward shock } \\
\hline & calculated (observed) & calculated (observed) & calculated (observed) \\
\hline$E_{\mathrm{m}, \mathrm{f}}(\mathrm{keV})$ & $330(\sim 100)$ & $3.2(\sim 10)$ & $10.13(\sim 50)$ \\
\hline$E_{\mathrm{c}, \mathrm{f}}(\mathrm{eV})$ & $591(-)$ & $962.5(-)$ & $141.7(-)$ \\
\hline$E_{\mathrm{m}, \mathrm{f}}^{(I C)}(\mathrm{GeV})$ & $2.46\left(\sim 30.5_{-2.6}^{+5.8}\right)$ & $16.3\left(\sim 33.4_{-3.5}^{+2.7}\right)$ & $9.15(\sim 10)$ \\
\hline$E_{\mathrm{c}, \mathrm{f}}^{(I C)}(e V)$ & $3.8 \times 10^{-2}(-)$ & $18.25 \times 10^{3}(-)$ & $18.4(-)$ \\
\hline Duration of the component (s) & $100(\sim 100)$ & $100(\sim 82)$ & $100(\sim 100)$ \\
\hline$\left(v F_{v \max }\right)^{S S C}\left(\mathrm{erg} \mathrm{cm}^{-2} s^{-1}\right)$ & $3.35 \times 10^{-5}\left(\sim 10^{-5}\right)$ & $2.33 \times 10^{-6}\left(\sim 10^{-6}\right)$ & $10.9 \times 10^{-7}\left(\sim 10^{-6}\right)$ \\
\hline \multicolumn{4}{|l|}{ Reverse shock } \\
\hline & calculated (observed) & calculated (observed) & calculated (observed) \\
\hline$E_{\mathrm{m}, \mathrm{r}}(\mathrm{keV})$ & $3.03(-)$ & $165.1(-)$ & $0.17(-)$ \\
\hline$E_{\mathrm{c}, \mathrm{r}}(\mathrm{eV})$ & $1.8 \times 10^{-3}(-)$ & $1.1 \times 10^{-3}(-)$ & $1.1 \times 10^{-3}(-)$ \\
\hline$E_{\mathrm{m}, \mathrm{r}}^{(I C)}(\mathrm{MeV})$ & $30.0 \times 10^{3}\left(10^{3}\right)$ & $414.3\left(10^{3}\right)$ & $414.3(400)$ \\
\hline$E_{\mathrm{c}, \mathrm{r}}^{(I C)}(e V)$ & $6.34 \times 10^{-7}(-)$ & $0.7 \times 10^{-5}(-)$ & $0.7 \times 10^{-5}(-)$ \\
\hline Duration of the component (s) & $6(<1)$ & $5(\sim 4)$ & $5(\sim 1)$ \\
\hline$\left(v F_{v \max }\right)^{S S C}\left(\mathrm{erg} \mathrm{cm}^{-2} \mathrm{~s}^{-1}\right)$ & $1.4 \times 10^{-6}\left(\sim 10^{-6}\right)$ & $8.47 \times 10^{-7}\left(\sim 10^{-6}\right)$ & $8.2 \times 10^{-6}\left(\sim 10^{-6}\right)$ \\
\hline
\end{tabular}

Table 1. Calculated quantities are given. For comparison, the corresponding observed values are given when available.

\section{References}

[1] C. D. Dermer \& A. Atoyan, Neutral beam model for the anomalous $\gamma$-ray emission component in GRB 941017 A\&A 418 (2004) L5

[2] C. D. Dermer \& S. Razzaque, Acceleration of Ultra-High-energy cosmic rays in the colliding shells of Bazars and GRB: Constraints from the Fermi gamma-ray space telescope ApJ 724 (2010) 1366

[3] H. Papathanassiou \& P. Meszaros, Spectra of Unsteady Wind Models of Gamma-Ray Bursts, ApJ 471 (1996) L91

[4] R. Sari, R. Narayan \& T. Piran, Cooling Timescales and Temporal Structure of Gamma-Ray Bursts, ApJ 473 (1996) 204

[5] M. Ackermann et al., Fermi Observations of GRB 090510: A Short-Hard Gamma-ray Burst with an Additional, Hard Power-law Component from 10 keV to GeV Energies, ApJ 716 (2010) 1178

[6] A. A. Abdo, et al., Fermi Observations of GRB 090902B: A Distinct Spectral Component in the Prompt and Delayed Emission, ApJ 706 (2009) L138

[7] M. Ackermann, et al., Detection of a Spectral Break in the Extra Hard Component of GRB 090926A, ApJ 729 (2011) 114

[8] R. Sacahui, N. Fraija , M. M. Gonzalez \& W. H. Lee 2012, The Long and the Short of the High-energy Emission in GRB090926A: An External Shock ApJ 755 (2012) 127

[9] N. Fraija, M. M. Gonzalez \& W. H. Lee 2012, Synchrotron Self-Compton Emission as the Origin of the Gamma-Ray Afterglow Observed in GRB 980923, ApJ 751 (2012) 33

[10] N. Fraija, M. M. Gonzalez \& W. H. Lee 2012, SSC Emission as Explanation of The Gamma Ray Afterglow Observed in GRB 980923, arXiv:1110.6421

[11] N. Fraija, M. M. Gonzalez, R. Sacahui, J. L. Ramirez, \& W. H. Lee In preparation 2012

[12] S. Golenetskii, et al., GRB Coordinates Network, 9959 (2009) 